ZU-TH 8/95

LMU-06/95

June 1995

\title{
Calculation of Long-Distance Effects in Exclusive Weak Radiative Decays of B-Mesons
}

\author{
A. Khodjamirian ${ }^{a, \dagger, 1}$, G. Stoll ${ }^{b}$, D. Wyler ${ }^{b}$ \\ ${ }^{a}$ Sektion Physik der Universität München \\ D-80333 München, Germany \\ ${ }^{b}$ Universität Zürich, 8057 Zürich, Switzerland
}

\begin{abstract}
We calculate the contribution of the weak annihilation to the $B \rightarrow \rho \gamma$ decay amplitude by means of QCD sum rules using the photon light-cone wave function. We find that this long-distance contribution amounts to about $10 \%$ of the leading short-distance effect in $B^{-} \rightarrow \rho^{-} \gamma$. On the other hand, weak annihilation is the dominant source of the corresponding $D$ meson decays and according to our estimates, yields branching ratios of $O\left(10^{-4}\right)$ for $D^{0} \rightarrow \bar{K}^{* 0} \gamma$, $O\left(10^{-5}\right)$ for $D_{s} \rightarrow \rho^{+} \gamma$, and $O\left(10^{-6}\right)$ for $D^{-} \rightarrow \rho^{-} \gamma$ and for $D^{0} \rightarrow \rho^{0} \gamma$.
\end{abstract}

${ }^{\dagger}$ on leave from Yerevan Physics Institute, 375036 Yerevan, Armenia

* partially supported by Schweizerischer Nationalfonds.

${ }^{1}$ supported by the German Federal Ministry for Research and Technology under contract No. 05 6MU93P 
1. Rare decays of $B$-mesons, such as the recently observed processes $B \rightarrow K^{*} \gamma$ and $B \rightarrow X_{s} \gamma$ [1, 2] are becoming an important tool for studying new forces beyond the standard model [3]. Indeed, many authors have investigated the effects of multiHiggs models, supersymmetric theories, left-right symmetric models, etc. on this process [4]. The interest in these decays stems from the fact that they occur only through loops and are therefore particularly sensitive to "new physics".

Besides the $b \rightarrow s$ transition, there are also $b \rightarrow d$ processes which are suppressed by the ratio $V_{t d} / V_{t s} \approx 0.1$ of CKM matrix elements. Within the standard model the rates are then estimated to be about $10^{-5}$ and $10^{-6}$ for $b \rightarrow d \gamma$ and $B \rightarrow \rho \gamma$, respectively. This mode is sensitive to other CKM matrix elements (and possibly to other new physics) and may show large CP-violation [5].

The "inclusive" processes like $b \rightarrow s \gamma$ can be usually evaluated on the quark level within perturbative methods [, but are more difficult experimentally. Exclusive processes, on the other hand are more easily observable, but require difficult nonperturbative calculations of matrix elements in order to yield useful results.

It is generally believed that the short distance penguin mechanism dominates the exclusive decays. The corresponding matrix element has been calculated with various methods [5, 6] which yield similar results; this indicates that the related uncertainties are presumably under control. Besides the matrix element of the penguin operator, there are other long-distance effects which must be analyzed. Several authors have recently investigated these effects [7, 8, 9, 10, 11, 12] in a phenomenological way. The crude estimates show that the long distance contributions can indeed be small. However, the results obtained vary enormously and can give at best an order of magnitude estimate. Clearly, a more reliable calculation is desired.

We may distinguish two long distance effects. First, there is a penguin type mechanism where the low momentum part of the usual penguin loop must be included. The above mentioned estimates so far indicate that it is at most several percent of the short distance result both for exclusive and inclusive processes. In these estimates, the radiative transition $B \rightarrow M \gamma$ is modelled by a sum over the processes $B \rightarrow M V^{*}$ followed by the transition $V^{*} \rightarrow \gamma$ where $V^{*}$ is a virtual vector meson with appropriate quantum numbers. The problems with this picture are obvious: the effective couplings (for the primary vertex and for the transition of the resonance into a photon) are only measured at the mass of the resonance and must be scaled to zero. Then, some information about the various structures of the primary vertex must exist, since only the transversal part can contribute to the photon transition. In the existing literature, one or the other of these problems is not addressed, but there is generally a strong suppression. For the particular case of $B \rightarrow K^{*} \gamma$ this suppression however, crucially depends on the model assumptions about the share of the factorizable contribution. If factorization dominates, we can combine the results of refs. [12] and [9] to predict a negligible effect for the $J / \Psi$ resonance. But only a more systematic calculation combined with direct estimate of nonfactorizable effects can clarify the role of this mechanism.

\footnotetext{
${ }^{1}$ An exception is the "long distance" part of the penguin mechanism (see below)
} 
Then, there is a completely different class of diagrams which do not involve loops of heavy (new) quarks but just the ordinary four fermion interaction. In these graphs, the 'spectator' participates in the weak annihilation; we therefore call them weak annihilation (WA). Because of the CKM matrix elements, this mechanism is negligible for $B \rightarrow K^{*} \gamma$ but important for other exclusive radiative decays. For definiteness, we will consider it for the decay $B \rightarrow \rho \gamma$, although other decays can be treated in similar ways and will be discussed briefly at the end. Fig. 1 represents schematically the mechanism. A perturbative evaluation of the diagrams in Fig.1 [7, 10 is rather uncertain, due to the almost on-shell propagation of the light quark which implies the use of a the poorly understood 'constituent' quark mass. The phenomenological description via the chain $B \rightarrow \rho \rho * \rightarrow \rho \gamma$ 10 suffers from the unknown $B \rho \rho$ coupling and the problems connected to the off-shellness of the $\rho$.

In this paper we show that existing QCD sum-rule technology allows us to provide reliable predictions for the long distance effects. We will discuss in this short note only the WA effects which are more uncertain in the phenomenological approach and which are important by themselves to a large class of transitions. We will only be interested in the leading effects; more accurate and further results will be presented in a future publication; we will asses here some of the remaining uncertainties.

2. The relevant effective Hamiltonian for $B \rightarrow \rho \gamma$ consists of two operators [13]:

$$
\mathcal{H}_{W}=\frac{G}{\sqrt{2}} V_{u b} V_{u d}^{*}\left\{\left(c_{1}\left(\bar{d} L_{\mu} u\right)\left(\bar{u} L^{\mu} b\right)+c_{2}\left(\bar{u} L_{\mu} u\right)\left(\bar{d} L^{\mu} b\right)\right\}\right.
$$

where $L_{\mu}=\gamma_{\mu}\left(1-\gamma_{5}\right)$, and $c_{1}$ and $c_{2}$ are the Wilson coefficients. For the decay $B^{-} \rightarrow \rho^{-} \gamma$ the combination

$$
\mathcal{H}_{W}=\frac{G}{\sqrt{2}} V_{u b} V_{u d}^{*} a_{1}\left(\bar{d} L_{\mu} u\right)\left(\bar{u} L^{\mu} b\right)
$$

with $a_{1}=c_{1}+c_{2} / 3$ enters. Similarly, the combination $a_{2}=c_{1} / 3+c_{2}$ multiplies the corresponding operator for $B^{0} \rightarrow \rho^{0} \gamma$. Following the phenomenological ansatz [14], the coefficients $a_{1}, a_{2}$ are extracted from two-body non-leptonic decays using factorization. Recent fits [15] indicate a considerable departure of $a_{2}$ from its short distance value (see, however, the recent discussion in [16]). The reason for that departure may be connected with nonfactorizable contributions to the hadronic matrix element, which in the decay $B^{-} \rightarrow \rho^{-} \gamma$ come from the color octet operator $\left(\bar{d} L_{\mu} \frac{\lambda^{a}}{2} u\right)\left(\bar{u} L^{\mu} \frac{\lambda^{a}}{2} b\right)$ with coefficient $2 c_{2}$.

The actual size of nonfactorizable effects is under discussion at present. First direct estimates [17], [18, 19] by QCD sum rule methods indicate that nonfactorizable effects in $B$ decays are quite process dependent. Moreover, it is not clear if one can use the same value of the effective parameters $a_{1,2}$ for nonleptonic and radiative 
decays, processes with different physical final states. Nevertheless, since the decay $B^{-} \rightarrow \rho^{-} \gamma$ depends on the parameter $a_{1}$ which is near its perturbative value $a_{1} \sim 1$, we assume for the present work that factorization is sufficient. At the same time one should keep in mind that the result for $B^{0} \rightarrow \rho^{0} \gamma$ which depends on $a_{2}$ will only be approximate.

Turning to more detailed analysis of the WA mechanism we first of all neglect the photon emission from the final state quarks invoking the well known helicity arguments: being factorized, the matrix element of (2) is in this case proportional to the light quark masses: $\left\langle\rho^{-}\left|H_{W}\right| B^{-}\right\rangle_{\gamma} \sim p_{B}^{\mu} f_{B}\left\langle\rho^{-}\left|\left(\bar{d} L^{\mu} u\right)\right| 0\right\rangle_{\gamma} \sim O\left(m_{u}, m_{d}\right)$. Note however that non-factorizable interactions such as gluon exchanges between final and initial quarks lift this suppression.

Thus we are left with two diagrams in Figs. 1a,b where the photon is emitted from the initial $b$ and $u$ quark lines. The corresponding matrix element can be written as

$$
\left\langle\rho^{-}\left|H_{W}\right| B^{-}\right\rangle_{\gamma}=\frac{G}{\sqrt{2}} V_{u b} V_{u d}^{*} a_{1} f_{\rho} m_{\rho} \epsilon_{\rho}^{\mu}\left\langle 0\left|\left(\bar{u} L_{\mu} b\right)\right| B^{-}\right\rangle_{\gamma}
$$

where we used $\left\langle\rho^{-}\left|\left(\bar{d} L^{\mu} u\right)\right| 0\right\rangle=f_{\rho} m_{\rho} \epsilon_{\rho}^{\mu}$, denoting by $f_{\rho}$ and $\epsilon_{\rho}$ the decay constant and the polarization vector of the charged $\rho$-meson. It then remains to calculate the matrix element

$$
\left\langle 0\left|\left(\bar{u} L^{\mu} b\right)\right| B^{-}\right\rangle_{\gamma}=-A_{P C} \varepsilon_{\mu \tau \lambda \sigma} p^{\tau} \epsilon^{\lambda} q^{\sigma}+i A_{P V}\left[q_{\mu}(\epsilon \cdot p)-\epsilon_{\mu}(p \cdot q)\right]
$$

which describes the annihilation of $B^{-}$into the current $\bar{u} L^{\mu} b$ with momentum $p$ accompanied by the emission of a real photon with momentum $q$ and polarization vector $\epsilon$. We have written explicitly decomposition of this matrix element into two gauge invariant (with respect to the electromagnetic field) terms $A_{P C}\left(A_{P V}\right)$ corresponding to parity conserving ( parity-violating) $B \rightarrow \rho \gamma$ decay amplitude. We note that for the short distance penguin mechanism one has $A_{P C}=A_{P V}$ if the light quark mass is neglected ( see e.g. [5]). In principle, polarization experiments could distinguish the two amplitudes and thus determine to what extent this equality is valid. In fact, it turned out that the WA mechanism considered here does not respect it, but the deviation is due to nonleading contributions and consequently small.

As noted, we propose to use QCD sum rules in order to calculate the matrix element (四). Since the photon emission from the light quark takes place at large distances, the use of standard QCD sum rules [20] based on the local operator product (OPE) expansion is not sufficient. Rather, one should use a light-cone expansion which is adequate for exclusive processes with light particles. It will involve the hadronic wave functions on the light-cone which encode the photon emission by a quark-antiquark pair at light-like separation in close analogy to the well known pion wave function [21]. The photon light-cone wave function was introduced in ref. 22 for calculating the amplitude of weak radiative decay $\Sigma \rightarrow \rho \gamma$ and used 
later in ref. [23 to evaluate the nucleon magnetic moments.

3. We now proceed to calculate the correlation function

$$
\Pi_{\mu}(p, q)=i \int d^{4} x e^{i p x}\left\langle 0\left|T\left\{\bar{u}(x) L_{\mu} b(x), \bar{b}(0) i \gamma_{5} u(0)\right\}\right| 0\right\rangle_{F(q)} .
$$

in the external electromagnetic field

$$
F_{\alpha \beta}(q, x)=i\left(\epsilon_{\beta} q_{\alpha}-\epsilon_{\alpha} q_{\beta}\right) e^{i q x}
$$

with momentum $q$ and polarization vector $\epsilon$. The function $\Pi_{\mu}$ can be decomposed into two parts corresponding to parity conserving and a parity violating part in (4):

$$
\Pi_{\mu}=\Pi_{P C} i \varepsilon_{\mu \tau \lambda \rho} p^{\tau} \epsilon^{\lambda} q^{\rho}+\Pi_{P V}\left[q_{\mu}(\epsilon \cdot p)-\epsilon_{\mu}(p \cdot q)\right]
$$

In the region $(p+q)^{2}<0$ and at $p^{2}=m_{\rho}^{2}<<m_{b}^{2}$, the heavy $b$-quark is far off-shell. In particular, photon emission from the heavy $b$-quark takes place perturbatively. The accompanying light-quark propagator may then be described by the local OPE containing as a first approximation the free propagation and in the next orders, interaction with quark and quark-gluon vacuum condensates. The corresponding contributions to the correlation function are depicted in Figs. 2a, b and c, respectively. As far as photon emission from the light $u$-quark is concerned, after contracting the $b$-quark line, one is left with matrix element

$$
\Pi_{\mu}(p, q)=i \int \frac{d^{4} x d^{4} k}{(2 \pi)^{4}\left(m_{b}^{2}-k^{2}\right)} e^{i(p-k) x}\left\langle 0\left|\bar{u}(x) L_{\mu}\left(m_{b}+\not k\right) \gamma_{5} u(0)\right| 0\right\rangle_{F(q)} .
$$

The diagram Fig. 2d describes only the short-distance part of this matrix element corresponding to the photon emission from freely propagating $u$ quark.

To take into account the long-distance part one needs to introduce additional nonperturbative parameters, describing the light-quark propagation in the presence of external electromagnetic field which is schematically shown in diagram of Fig. 2e. In the case of constant electromagnetic field $(q \rightarrow 0)$ one still can use the local OPE i.e. expand (8) around $x=0$ in the external field [24, 25]. Physically, the most important parameter emerging in this expansion is the so called induced quark condensate

$$
\left\langle 0\left|\bar{q} \sigma_{\alpha \beta} q\right| 0\right\rangle_{F}=e_{q}\langle\bar{q} q\rangle \chi F_{\alpha \beta}
$$

where $e_{q}$ is the quark electric charge, $\langle\bar{q} q\rangle$ is the quark condensate density and parameter $\chi$ has physical meaning of magnetic susceptibility of the quark condensate.

However, here we deal with essentially large photon momenta of the order of heavy quark mass. In this kinematical configuration the use of local OPE will lead to unmanageable infinite series of condensates for the expansion of (8) (see a 
recent detailed discussion in [26]). The adequate approach is to use the expansion near the light-cone $x^{2}=0$ introducing matrix elements of nonlocal operators. The leading twist two contribution [22, 23] emerges from the expansion of the operator $\bar{u}(x) \sigma_{\alpha \beta} u(0)$ and the corresponding part of the matrix element in the external photon field can be parametrized as 2

$$
\left\langle 0\left|\bar{u}(x) \sigma_{\alpha \beta} u(0)\right| 0\right\rangle_{F(q)}=e_{u}\langle\bar{u} u\rangle \int_{0}^{1} d u \varphi(u) F_{\alpha \beta}(u x) \text {. }
$$

Here, the function $\varphi(u)$ has the meaning of the photon wave function in terms of its quark-antiquark constituents and may be interpreted as the distribution in the fraction of light-cone momentum $q_{0}+q_{3}$ of the photon carried by a quark. The asymptotic form of this wave function is known [21, 22]:

$$
\varphi(u)=6 \chi u(1-u)
$$

where the appearance of the magnetic susceptibility $\chi$ is evident from taking local limit of (10) and comparing with the definition (9). Moreover, according to the analysis carried out in ref. [22], non-asymptotic effects in $\varphi(u)$ and higher twist (twist 4) contributions to (10) are small, contrary to the case of the pion wave functions. Therefore, in what follows we will use the asymptotic wave function (11). A rough estimate of the twist four contribution carried out below will allow us to neglect all higher twist effects.

To proceed, we retain in eq. (8) the parts containing $\bar{u}(x) \sigma_{\mu \nu} u(0)$, insert eq. (10), and after integration over $x$ and $k$ obtain the leading twist 2 contribution to the correlation function (5) corresponding to the long distance photon emission from u-quark and depicted in Fig. 2e:

$$
\Pi_{P C}^{t w i s t 2}=\Pi_{P V}^{t w i s t 2}=i e_{u}\langle\bar{u} u\rangle \int_{0}^{1} d u \frac{\varphi(u)}{m_{b}^{2}-(p+u q)^{2}} .
$$

The next important contribution stems from the perturbative loop diagrams of Figs. 2a and $2 \mathrm{~d}$. Their contribution to the parity conserving invariant amplitude written in the form of dispersion integral in variable $(p+q)^{2}$ at fixed $p^{2}$ is:

$$
\Pi_{P C}^{p e r t}=\frac{3 i m_{b}}{4 \pi^{2}} \int_{m_{b}^{2}}^{\infty} \frac{d s}{\left[s-(p+q)^{2}\right]\left(s-p^{2}\right)}\left[\left(e_{u}-e_{b}\right) \frac{s-m_{b}^{2}}{s}+e_{b} \ln \frac{s}{m_{b}^{2}}\right]
$$

where the pieces proportional to $e_{b}$ and $e_{u}$ correspond to the diagrams Fig. $2 \mathrm{a}$ and $2 \mathrm{~d}$, respectively. The contribution of the loop diagrams to the parity violating amplitude is:

$$
\Pi_{P V}^{p e r t}=\frac{3 i m_{b}}{4 \pi^{2}} \int_{m_{b}^{2}}^{\infty} \frac{d s}{\left[s-(p+q)^{2}\right]\left(s-p^{2}\right)^{2}}\left[\left\{e_{u}\left(2 m_{b}^{2}-s-\frac{p^{2} m_{b}^{2}}{s}\right)\right.\right.
$$

\footnotetext{
2 the path-ordered exponential gauge factors for both gluon and photon fields are not shown for brevity
} 


$$
\left.\left.-e_{b}\left(2 m_{b}^{2}-s-\frac{p^{2}\left(m_{b}^{2}-2 s\right)}{s}\right)\right\} \frac{s-m_{b}^{2}}{s}+e_{b}\left(2 m_{b}^{2}-s+p^{2}\right) \ln \frac{s}{m_{b}^{2}}\right] .
$$

We see that the amplitudes $\Pi_{P C}$ and $\Pi_{P V}$ are indeed different. To assess the importance of nonperturbative effects in the case when photon is emitted from the heavy quark we have also calculated the contributions of quark condensate corresponding to Fig. $2 \mathrm{~b}$ with the result

$$
\Pi_{P C}^{\langle\bar{q} q\rangle}=\Pi_{P V}^{\langle\bar{q} q\rangle}=-i e_{b} \frac{\langle\bar{u} u\rangle}{\left(m_{b}^{2}-p^{2}\right)\left(m_{b}^{2}-(p+q)^{2}\right)},
$$

In calculating the perturbative and quark condensate contributions, the contact terms violating gauge invariance with respect to the photon field should be properly taken into account. If one carries out the calculation in the fixed point gauge for the photon field, the origin must be chosen carefully. The quark-gluon condensate contribution (Fig. 2c) and higher-twist contributions corresponding to three-particle photon wave function (Fig. 2f) are neglected in the present calculation.

To understand the level of accuracy for the most important contribution containing the photon wave function we need some estimate of nonleading higher twist contributions neglected in the matrix element in eq. (10). We parametrize the next-to-leading twist 4 contribution in the expansion of this matrix element as :

$$
\begin{array}{r}
\left\langle 0\left|\bar{u}(x) \sigma_{\alpha \beta} u(0)\right| 0\right\rangle_{F(q)}^{t w i s t 4}=e_{u}\langle\bar{u} u\rangle \int_{0}^{1} d u\left\{f_{1}(u) F_{\alpha \beta}(u x) x^{2}\right. \\
\left.+f_{2}(u)\left[F_{\alpha \rho}(u x) x_{\rho} x_{\beta}-F_{\beta \rho}(u x) x_{\rho} x_{\alpha}\right]\right\} .
\end{array}
$$

As a result,

$$
\Pi_{P C}^{t w i s t 4}=-4 i e_{u}\langle\bar{u} u\rangle \int_{0}^{1} d u \frac{f_{1}(u)}{\left[m_{b}^{2}-(p+u q)^{2}\right]^{2}}\left[1+\frac{2 m_{b}^{2}}{m_{b}^{2}-(p+u q)^{2}}\right]
$$

and the corresponding answer for $\Pi_{P V}^{t w i s t 4}$ is obtained by replacing $f_{1}(u)$ in the numerator of (17) by the combination $f_{1}+f_{2}$. The only information about distributions $f_{1,2}$ is available from comparison with the twist 4 terms in the light-cone sum rules for the $\Sigma \rightarrow p \gamma$ decay [22] and for the nucleon magnetic moment 23] which result in the following relation:

$$
f_{1}(u)+\frac{f_{2}(u)}{2}=-\frac{1}{4}\left[(1-u)-\varphi_{4}(u)\right] .
$$

The first term in r.h.s. of (18) originates from the expansion of the light quark field in the electromagnetic field, whereas the wave function $\varphi_{4}$ corresponds to analogous expansion in the gluon field and is related to the three-particle photon wave functions of twist four via QCD equation of motion. According to estimates of [22], $\varphi_{4}(u) \simeq$ $30 k u^{2}(1-u)^{2}+\ldots$, with the parameter $k \simeq-0.2$ and the nonasymptotic corrections denoted by ellipses and not shown for brevity. We assume for an order of magnitude estimate that $f_{1}=f_{2}$, having in mind that the normalization integrals of these two 
distributions are equal. We plan to analyze this contribution in more details in future.

4. The QCD sum rule is obtained as usual by equating the hadronic representation of the correlation function $\Pi_{\mu}$ with the result of the QCD calculation, a sum of all contributions calculated above. Inserting hadronic states with $B$ meson quantum numbers into eq.(5), we have the following decomposition for invariant amplitudes in eq.(可):

$$
\Pi_{P C(P V)}=\frac{i f_{B} m_{B}^{2} A_{P C(P V)}}{m_{b}\left[m_{B^{2}}-(p+q)^{2}\right]}+\int_{s_{0}}^{\infty} d s \frac{\rho_{P C(P V)}^{h}\left(s, p^{2}\right)}{s-(p+q)^{2}}
$$

where the first term represent the $B$-meson contribution containing the matrix element (4) and $\left\langle B\left|\bar{b} i \gamma_{5} u\right| 0\right\rangle=f_{B} m_{B}^{2} / m_{b}$. The second term in (19) represents contribution of the higher states in $B$ channel starting from some effective threshold $s_{0}$. We invoke the usual hadron-quark duality and replace the spectral density $\rho_{P C(P V)}^{h}$ by the imaginary part of $\Pi_{P C(P V)}$ calculated in QCD. Equating now eq. (19) with the result of this calculation given in eqs. (12)-(15) and applying a Borel transformation in $(p+q)^{2}$ to suppress the higher states we obtain the desired QCD sum rule for both amplitudes entering the hadronic matrix element (4). The result for the parity conserving amplitude is

$$
\begin{gathered}
A_{P C}=\frac{m_{b}}{f_{B} m_{B}^{2}}\left\{\int _ { \Delta } ^ { 1 } \frac { d u } { u } \operatorname { e x p } [ \frac { m _ { B } ^ { 2 } } { M ^ { 2 } } - \frac { m _ { b } ^ { 2 } - p ^ { 2 } ( 1 - u ) } { u M ^ { 2 } } ] \left[e_{u}\langle\bar{u} u\rangle \varphi(u)\right.\right. \\
\left.+\frac{3 m_{b}}{4 \pi^{2}}\left(\left(e_{u}-e_{b}\right) \frac{\left(m_{b}^{2}-p^{2}\right)(1-u)}{m_{b}^{2}-p^{2}(1-u)}+e_{b} \ln \left[\frac{m_{b}^{2}-p^{2}(1-u)}{u m_{b}^{2}}\right]\right)\right] \\
\left.-\frac{e_{b}\langle\bar{u} u\rangle}{m_{b}^{2}-p^{2}} \exp \left(\frac{m_{B}^{2}-m_{b}^{2}}{M^{2}}\right)\right\},
\end{gathered}
$$

and for the parity violating amplitude

$$
\begin{aligned}
& A_{P V}=\frac{m_{b}}{f_{B} m_{B}^{2}}\left\{\int _ { \Delta } ^ { 1 } \frac { d u } { u } \operatorname { e x p } [ \frac { m _ { B } ^ { 2 } } { M ^ { 2 } } - \frac { m _ { b } ^ { 2 } - p ^ { 2 } ( 1 - u ) } { u M ^ { 2 } } ] \left[e_{u}\langle\bar{u} u\rangle \varphi(u)\right.\right. \\
&+ \frac{3 m_{b}}{4 \pi^{2}}\left(\frac{m_{b}^{2}}{m_{b}^{2}-p^{2}}\right)\left\{\left[e_{u}\left(2 u-1+\frac{p^{2}(1-u)}{m_{b}^{2}}-\frac{p^{2} u^{2}}{m_{b}^{2}-p^{2}(1-u)}\right)\right.\right. \\
&\left.-e_{b}\left(2 u-1+\frac{p^{2}(1+u)}{m_{b}^{2}}-\frac{p^{2} u^{2}}{m_{b}^{2}-p^{2}(1-u)}\right)\right] \frac{\left(m_{b}^{2}-p^{2}\right)(1-u)}{m_{b}^{2}-p^{2}(1-u)} \\
&\left.\left.\left.+e_{b} \ln \left[\frac{m_{b}^{2}-p^{2}(1-u)}{u m_{b}^{2}}\right]\left(2 u-1+\frac{p^{2}}{m_{b}^{2}}\right)\right\}\right]-\frac{e_{b}\langle\bar{u} u\rangle}{m_{b}^{2}-p^{2}} \exp \left(\frac{m_{B}^{2}-m_{b}^{2}}{M^{2}}\right)\right\}
\end{aligned}
$$

where $M^{2}$ is the Borel parameter.

In order to compactify these expressions, the dispersion integrals for the loop contributions are written in terms of a new integration variable $u=\left(m_{b}^{2}-p^{2}\right) /\left(s-p^{2}\right)$, so that substitution of $\Delta=\left(m_{b}^{2}-p^{2}\right) /\left(s_{0}-p^{2}\right)$ instead of the lower limit in all 
integrals in $(20), 21)$ is equivalent to subtraction of higher state contributions. Note that the results of our calculation are, in general, the two form factors $A_{P C, P V}\left(p^{2}\right)$ determining $p^{2}$ dependence of the matrix element (4). For the problem under investigation we need only the values of these form factors at $p^{2}=m_{\rho}^{2}$ or at $p^{2}=m_{K^{*}}^{2}$. The sum rules are valid in a much wider region of timelike variable $p^{2}$, parametrically up to $O\left(m_{b}^{2}-O\left(\mathrm{GeV}^{2}\right)\right.$ ) and practically up to $p^{2}=15 \mathrm{GeV}^{2}$ (see analysis of analogous sum rules for heavy-to-light form factors in [6, 26, 27]).

5. We proceed now with the numerical analysis. The important point is that all parameters entering the l.h.s of eqs. (20), (21) are known since they also enter various other QCD sum rules; the universality of nonperturbative inputs is of course the main advantage of this approach. The value of magnetic susceptibility was determined several times [28], 229] with essentially the same result $\chi=-4.4 \mathrm{GeV}^{-2}$ at the normalization scale of $1 \mathrm{GeV}$. We use $\langle\bar{u} u\rangle=-(240 \mathrm{MeV})^{3}$ at this scale. The anomalous dimension of the $\bar{q} \sigma_{\mu \nu} q$ operator is known [30] to be $(-4 / 27)$ whereas the anomalous dimension of the quark condensate operator is (4/9) resulting in $\chi=-3.4 \mathrm{GeV}^{-2}$ and $\langle\bar{u} u\rangle=-(260 \mathrm{MeV})^{3}$ at the scale $\mu_{b} \sim \sqrt{m_{B}^{2}-m_{b}^{2}}$ inherent to the correlation function (5). The comparatively large numerical value of the parameter $\chi$ justifies the use of leading twist approximation. The relatively high scale $\mu_{b}$ is an additional argument on favor of the asymptotic form (11) throughout our numerical calculation.

The values of all parameters corresponding to the $B$-channel are known from two-point sum rules for the b-quark current: $f_{B}=140 \mathrm{MeV}, m_{b}=4.7 \mathrm{GeV}, s_{0}=$ $35 \mathrm{GeV}^{2}$. (see e.g. 26, 27]). Note that the $B$-meson decay constant is taken without $O\left(\alpha_{s}\right)$ corrections since they are also not included in the sum rules (20) and (21) where they partially cancel those of $f_{B}$. With these parameters, the amplitudes $A_{P C}$, $A_{P V}$ are calculated at $p^{2}=m_{\rho}^{2}$ as a function of the Borel parameter. The predictions of the sum rules are very stable in a rather wide region of Borel parameter and vary by at most $5 \%$ with the changes of $m_{b}, s_{0}, f_{B}$ within the intervals allowed by the two-point sum rule for $f_{B}$. In analogy with other sum rules we isolate a certain interval of Borel parameter which we determine as $8<M^{2}<12 \mathrm{GeV}^{2}$. Here our estimate of twist four operators gives contributions below the $5 \%$ level and higher states according to the quark-hadron duality model contribute less than $30 \%$. Our final prediction for the amplitudes defining the matrix element (4) for the WA decay $B^{-} \rightarrow \rho^{-} \gamma$ is :

$$
A_{P C}\left(B^{-} \rightarrow \rho^{-} \gamma\right)=1.1 \cdot 10^{-2} \mathrm{GeV}^{-1}, \quad A_{P V}\left(B^{-} \rightarrow \rho^{-} \gamma\right)=0.85 \cdot 10^{-2} \mathrm{GeV}^{-1}
$$

with negligible variation within the interval of $M^{2}$ specified above. The corresponding prediction for the amplitude of the neutral mode $\bar{B}^{0} \rightarrow \rho^{0} \gamma$ is easily obtained 
by replacing $u \rightarrow d$ in the sum rules. The prediction in the same Borel window is :

$$
A_{P C}\left(\bar{B}^{0} \rightarrow \rho^{0} \gamma\right)=-7 \cdot 10^{-3} \mathrm{GeV}^{-1}, \quad A_{P V}\left(\bar{B}^{0} \rightarrow \rho^{0} \gamma\right)=-5 \cdot 10^{-3} \mathrm{GeV}^{-1}
$$

All these predictions have a negligible variation with the Borel parameter within the interval specified above. As expected, an important part of the amplitudes (22) and (23), $(40 \div 50) \%$ comes from the contribution of the photon twist-two wave function. The contribution from the loop diagram with photon emission from the light quark is about the same and amounts to $(50 \div 60) \%$ of the total result. The photon emission from the heavy quark (both perturbative and nonperturbative) is negligibly small. The strong isospin violation (or in other words, dependence on the flavour of the spectator quark) which is evident from the ratios of the amplitudes of charged (22) and neutral (23) modes, is one of the characteristic features of the WA mechanism in contrast to the short distance penguin amplitudes which are independent of the flavour of the spectator quark.

Concerning the accuracy of our calculation we first notice that omitted higher order terms are indeed very small. The higher order quark-gluon condensate contribution, Fig. 2c, can be safely neglected since the quark condensate contribution itself turns out to be at the level of $1 \%$. Also the estimate of twist 4 contribution allows to neglect the diagram Fig. $2 \mathrm{f}$ containing the twist 4 three-particle photon wave function. One may even argue that this diagram is additionally suppressed because the gluon is emitted from the heavy quark line (such a suppression is present in other light-cone sum rules for the heavy-to light transition amplitudes, see e.g. [27, 26] ). The higher twist terms and nonasymptotic corrections are the main source of uncertainty in the light-cone sum rules involving the pion wave functions. In the present situation, the main uncertainty comes from the range of the parameter $\chi$ which is known with $10 \%$ accuracy. Our conservative estimate of the overall accuracy is about $15-20 \%$. Furthermore, there are $O\left(\alpha_{s}\right)$ corrections to the correlation function (5). Presumably, the largest part originates from perturbative gluon exchanges in the leading diagram of Fig. 2e. Their calculation is straightforward, but beyond the goal of our work.

It is immediate to convert eqs. (20) and (21) into the sum rules for the analogous hadronic matrix elements $\left\langle 0\left|\left(\bar{d}(\bar{u}) L^{\mu} c\right)\right| D^{+(0)}\right\rangle_{\gamma}$ determining the radiative decays of charmed mesons $D \rightarrow \rho \gamma$ via WA mechanism. One has just to replace $b$ with $c$, B with D in the sum rules (20), (21) and substitute corresponding parameters: $f_{D}=170 \mathrm{MeV}, m_{c}=1.3 \mathrm{GeV}, s_{0}=6 \mathrm{GeV}^{2}$, (we take them from 26]) $\chi=-4.0$ $\mathrm{GeV}^{-2}$ and $\langle\bar{u} u\rangle=-(250 \mathrm{MeV})^{3}$.

In the Borel parameter range $3.0<M^{2}<4.5 \mathrm{GeV}^{2}$ which satisfy all usual requirements we obtain (with small variations)

$$
\begin{aligned}
& A_{P C}\left(D^{+} \rightarrow \rho^{+} \gamma\right)=-1.7 \cdot 10^{-2} \mathrm{GeV}^{-1}, \quad A_{P V}\left(D^{+} \rightarrow \rho^{+} \gamma\right)=-1.5 \cdot 10^{-2} \mathrm{GeV}^{-1} \\
& A_{P C}\left(D^{0} \rightarrow \rho^{0} \gamma\right)=+9.6 \cdot 10^{-2} \mathrm{GeV}^{-1}, \quad A_{P V}\left(D^{0} \rightarrow \rho^{0} \gamma\right)=+5.5 \cdot 10^{-2} \mathrm{GeV}^{-1}
\end{aligned}
$$

For the correlation function (5) with $c$ quark currents the hierarchy of contributions drastically changes. The magnitude of photon emission from the $c$ quark is almost 
as high as from the light quark. At the same time the relative role of the nonperturbative emission from the light quark (i.e. of the photon wave function contribution) compared with the perturbative emission is larger. As a result, the amplitudes (24) and (25) represent interferences of various important contributions and the role of subleading terms is in general higher, especially for $D^{-}$decay where the estimate of the size of the twist 4 term indicates a 20\% correction. For this particular mode a higher accuracy is required, including the calculation of terms omitted in our present calculation.

For completeness we list the corresponding amplitudes for the remaining $B$ and $D$ modes which are simply connected to the modes considered above :

$$
A\left(B^{-} \rightarrow K^{*-} \gamma\right) \simeq A\left(B^{-} \rightarrow \rho^{-} \gamma\right)
$$

and

$$
A\left(D^{0} \rightarrow \bar{K}^{* 0} \gamma\right) \simeq A\left(D^{0} \rightarrow \rho^{0} \gamma\right), A\left(D^{+} \rightarrow \rho^{+} \gamma\right) \simeq A\left(D_{s} \rightarrow \rho^{+} \gamma\right) .
$$

These relations are valid for both $P C$ and $P V$ amplitudes in the $\mathrm{SU}(3)$ flavour limit, i.e. if one can neglect the variation of form factors $A_{P C, P C}$ from $p^{2}=m_{\rho}^{2}$ to $p^{2}=m_{K^{*}}^{2}$ or, in the case of the second relation in (27), the difference between photon emission from $s$ and $d$ quarks. From our sum rules we are able to safely predict that the amplitudes at the point $p^{2}=m_{K^{*}}^{2}$ are $O(1 \%)(O(10 \%))$ higher for $B(D)$. The WA mechanism is absent for the modes $B^{0} \rightarrow \bar{K}^{* 0} \gamma, B_{s} \rightarrow K^{* 0} \gamma, B_{s} \rightarrow \phi \gamma$. and for $D^{-} \rightarrow \bar{K}^{*-} \gamma$ ) (with the possible exception of CKM suppressed contributions).

6. With the amplitudes determining the heavy meson annihilation matrix element (4), we can finally compare the WA effects to that of the short-distance penguin mechanism and obtain the corrections to the branching ratios.

The branching ratio corresponding to the decay amplitude (3) with the photon transition matrix element defined in (雨) is:

$$
B R\left(B^{-} \rightarrow \rho^{-} \gamma\right)=\frac{G^{2}}{64 \pi}\left(V_{u b} V_{u d}^{*} a_{1}\right)^{2} f_{\rho}^{2} m_{\rho}^{2}\left(\frac{m_{B}^{2}-m_{\rho}^{2}}{m_{B}}\right)^{3}\left[A_{P C}^{2}+A_{P V}^{2}\right] \tau_{B^{-}}
$$

for $B^{-} \rightarrow \rho^{-} \gamma$ and with obvious substitutions for the other decays. Taking for the present analysis $a_{1}=1, f_{\rho}=216 \mathrm{MeV}$ from the leptonic width of $\rho$-meson which we take along with all other parameters entering (28) from [31] we finally obtain for the branching ratio corresponding to the WA mechanism :

$$
B R\left(B^{-} \rightarrow \rho^{-} \gamma\right) \simeq 7.0 \cdot 10^{-5} \mathrm{GeV}^{2}\left(\frac{V_{u b}}{0.0035}\right)^{2}\left(\frac{a_{1}}{1.0}\right)^{2}\left[A_{P C}^{2}+A_{P V}^{2}\right]
$$

Here uncertainties connected with the weak interaction vertex are emphasized and our choice for the corresponding most uncertain parameters is shown explicitly. We 
recall once again that the estimate (28) depends on the validity of the factorization approximation, which in particular, allows to neglect the photon emission from final state quarks.

In order to compare our resluts for the WA mechanism to the short distance (SD) penguin contribution, we cast the latter into the form of eq. (29). Using the results of [5, 6] we obtain

$$
\begin{aligned}
& \left|A_{P C}\left(B^{-} \rightarrow \rho^{-} \gamma\right)^{S D}\right|=\left|A_{P V}\left(B^{-} \rightarrow \rho^{-} \gamma\right)^{S D}\right| \\
& \simeq 0.12 \mathrm{GeV}^{-1}\left(\frac{\left|V_{t d}\right|}{0.0097}\right)\left(\frac{0.0035}{\left|V_{u b}\right|}\right)\left(\frac{1.0}{a_{1}}\right) .
\end{aligned}
$$

Thus, our result in eq. (22) amounts to about a $10 \%$ correction to the SD amplitude for $B^{-} \rightarrow \rho^{-} \gamma$ with uncertainty at the level of $50 \%$ from the values of the CKM matrix elements. This correction is comparable to the present accuracy of the hadronic matrix elements in refs. [5, 6]; with more refined techniques, however, the WA contributions should presumably not be neglected in predicting the branching ratio of $B^{-} \rightarrow \rho^{-} \gamma$ and the $\mathrm{CP}$-violating asymmetries [5]. In comparison, the phenomenological estimates of the long distance penguin contributions [9, 12] indicate that these are quite small and probably negligible. The correction of WA mechanism in $\bar{B}^{0} \rightarrow \rho^{0} \gamma$ turns out to be much smaller, at the level of $1 \%$, due to relative factor $a_{2} / a_{1}$ and due to relative smallness of the amplitudes (23) as compared with (22).

Unlike in radiative $B$-decays, the WA effects are crucial in the corresponding $D$ decays. The short distance penguin contributions are completely negligible, while the long distance terms may even lead to observable rates 11, 32, 33:

$$
B R\left(D^{+} \rightarrow \rho^{+} \gamma\right)=\frac{G^{2}}{64 \pi}\left(V_{c d} V_{u d}^{*} a_{1}\right)^{2} f_{\rho}^{2} m_{\rho}^{2}\left(\frac{m_{D}^{2}-m_{\rho}^{2}}{m_{D}}\right)^{3}\left[A_{P C}^{2}+A_{P V}^{2}\right] \tau_{D^{-}}
$$

Using the values in eqs.(24) and (25), we obtain

$$
B R\left(D^{+} \rightarrow \rho^{+} \gamma\right) \simeq 2.7 \cdot 10^{-6}, \quad B R\left(D^{0} \rightarrow \rho^{0} \gamma\right) \simeq 3.1 \cdot 10^{-6}\left(a_{2} / 0.5\right)^{2} .
$$

With the relations (27) we predict the branching ratio for $D^{0} \rightarrow \bar{K}^{* 0} \gamma$ to be $1.5 \cdot 10^{-4}\left(a_{2} / 0.5\right)^{2}$ and for $D_{s} \rightarrow \rho^{+} \gamma$ to be $2.8 \cdot 10^{-5}$.

We can apply the present technology to the decays $B \rightarrow l \nu_{l} \gamma$ [34]; we simply replace the $\rho$ current with the leptonic one. With the notation of ref. [35], we obtain

$$
R_{B}^{\mu}=\frac{1}{24 \pi^{2} f_{B}^{2} m_{\mu}^{2} m_{B}^{4}} \int_{0}^{m_{B}^{2}} d p^{2}\left(m_{B}^{2}-p^{2}\right)^{3} p^{2}\left\{A_{P C}^{2}\left(p^{2}\right)+A_{P V}^{2}\left(p^{2}\right)\right\}
$$

for the ratio between the purely leptonic decay modes with and without photon. A problem arises because we now need the form factor for all values of $p^{2}$ and not just at the $\rho$ mass, and the sum rule method becomes unreliable for large $p^{2}$ values. We can overcome the difficulty by assuming a pole behaviour (with $B^{*}$ pole for $A_{P C}$ and axial $B$ pole for $A_{P V}$ ) and normalizing it with our numbers obtained from the sum 
rules (20) and (21) at $p^{2}=15 G e V^{2}$. The phase space suppression at large values of the lepton pair invariant mass makes the result insensitive to the accuracy of this extrapolation. We then obtain $R_{B}^{\mu} \simeq 19.0$ which is in agreement with the earlier work [35, 36] and underlines the importance of the radiative leptonic decays.

Finally, we should mention that the same correlation function (5) may be used to derive the sum rule for the $B^{*} B \gamma$ and $D^{*} D \gamma$ couplings. The derivation follows essentially the same steps as in [26] where the light-cone sum rules for the $B^{*} B \pi$ and $D^{*} D \pi$ couplings have been obtained. The detailed analysis is beyond the scope of the present investigation and will be presented in a separate paper [37. We just mention here that our preliminary results on the photonic $D^{*}$ width combined with its pionic widths obtained in 26] agree well with experimental data.

7. In this paper we have calculated a part of the long-distance contributions to exclusive radiative rare $B$ and $D$ decays. We have shown that the QCD sum rules with the light-cone photon wave function allow to calculate them rather reliably. In the case of the decays $B \rightarrow \rho \gamma$ the long-distance contributions considered are small, especially in the neutral mode, which allows to neglect them in the determination of $V_{t d}$ through that decay. We predict branching ratios for the corresponding $D$ decays where the long-distance weak annihilation dominates. There are other nonperturbative effects which can be estimated in a similar fashion; work on this is in progress.

\section{Acknowledgements.}

We thank D. Atwood, V. Braun, B.L. Ioffe, M. Misiak, M. Petermann, R. Rückl and A. Soni for very useful and stimulating discussions.

\section{References}

[1] R. Ammar et al (CLEO Collaboration), Phys. Rev. Lett. 71 (1993) 674.

[2] M.S. Alam et al ( CLEO Collaboration), preprint CLNS 94-1314 (1994).

[3] A.J. Buras, M. Misiak, M. Münz and S. Pokorski, Nucl. Phys. B424 (1994) 374.

[4] J. Hewett, Preprint SLAC-PUB-6521 (1994);

J. Milana, Phys. Rev. D51 (1995) 1192.

[5] C. Greub, H. Simma and D. Wyler, Nucl. Phys. B147 (1995) 434. For earlier references on the hadronic radiative rates see D. Wyler, Nucl. Phys B. (Proc. Suppl.) 7 A (1989).

[6] A. Ali, V.M. Braun and H. Simma, Z. Phys. C63 (1994) 437. 
[7] H.-Y.Cheng et. al. Phys. Rev. D51 (1995) 1199;

H.-Y. Cheng, Preprint IP-ASTP-11-94, hep-ph/940626.

[8] E. Golowich and S. Pakvasa, Phys. Rev. D51 (1995) 1215.

[9] N. Deshpande, X.-G. He and J. Trampetic, preprint OITS-564, hep-ph/9412222.

[10] D. Atwood, B. Blok and A. Soni, preprint SLAC-PUB-6635, hep-ph/ 9408373.

[11] G. Burdman et. al., preprint FERMILAB-Pub-94/412-T, hep-ph/9502329.

[12] J. Soares, preprint TRI-PP-95-6, hep-ph/9503285.

[13] M.K. Gaillard and B. W. Lee, Phys. Rev. Lett. 33 (1974) 108;

G. Altarelli and L.Maiani, Phys. Lett. B52 (1974) 351;

A. Buras and P. Weisz, Nucl. Phys. B333 (1990) 66.

[14] M. Wirbel, B. Stech and M. Bauer, Z. Phys. C29 (1985) 637; C34 (1987) 103.

[15] M.S. Alam et al.( CLEO Collaboration) Phys. Rev. D50 (1994) 43 .

[16] A.J. Buras, Nucl. Phys. B434 (1995) 606.

[17] B. Blok, M. Shifman, Sov. J. Nucl. Phys. 45 (1987) 135, 301, 522; Nucl. Phys. B389 (1993) 534 .

[18] A. Khodjamirian, R. Rückl, Proc. Workshop 'Continuous Advances in QCD' , ed. A.V. Smilga, World Scientific (1994) p.287;

A. Khodjamirian, B. Lampe, R. Rückl in preparation.

[19] I. Halperin, preprint TECHNION-PHYS-94-16, hep-ph/9411422.

[20] M.A. Shifman, A.I. Vainshtein and V.I. Zakharov, Nucl. Phys. B147 (1979) $385,448$.

[21] V.L. Chernyak and A.R. Zhitnitsky, JETP Lett. 25 (1977) 510; Yad. Fiz. 31 (1980) 1053; Phys. Rep. 112 (1984) 173.

A.V. Efremov and A.V. Radyushkin, Phys. Lett. B94 (1980) 245; Teor. Mat. Fiz. 42 (1980) 147.

G.P. Lepage and S.J. Brodsky, Phys. Lett. B87 (1979) 359; Phys. Rev. D22 (1980) 2157.

[22] I.I. Balitsky, V.M. Braun and A.V. Kolesnichenko, Sov. J. Nucl. Phys. 44 (1986) 1028; Nucl. Phys. B312 (1989) 509.

[23] V.M. Braun and I.B. Filyanov, Z. Phys. C 44 (1989) 157. 
[24] B.L. Ioffe and A.V. Smilga, Pisma v ZheTF, 37 (1983) 230; Nucl. Phys. B232 (1984) 109.

[25] Ya. Balitsky and A.V. Yung Phys. Lett. B129 (1983) 328.

[26] V.M. Braun, V.M. Belyaev, A. Khodjamirian and R. Rückl, to be published in Phys. Rev. D , hep-ph/9410280.

[27] V.M. Belyaev, A. Khodjamirian and R. Rückl, Z. Phys. C60 (1993) 349.

[28] V. M Belyaev and Ya. I. Kogan, Sov. J. Nucl. Phys. 40 (1984) 659.

[29] I.I. Balitsky, A.V. Kolesnichenko and A.V. Yung, Sov. J. Nucl. Phys. 41 (1985) 178 ;

C.B. Chiu , J. Pasupathy and S.J. Wilson, Phys. Rev. D33 (1986) 1961.

[30] M.A. Shifman and M.I. Vysotsky, Nucl. Phys. B186 (1981) 475.

[31] Particle Data Group, Phys. Rev. D50 (1994) 1.

[32] K. Babu et al. Phys. Lett. B205 (1988) 540;

S. Egli et al. in proc. DESY HERA Workshop Eds. W. Buchmüller and G. Ingelman (1991) p.770.

[33] B. Bajc, S. Fajfer, R.J. Oakes, Phys. Rev. D51 (1995) 2230.

[34] we thank D. Atwood for suggesting this decay to us.

[35] G. Burdman, T. Goldman and D.Wyler, Phys. Rev. D51 (1995) 1995.

[36] D. Atwood, G. Eilam and A. Soni, preprint SLAC-PUP-95-6716, hep-ph/9411367.

[37] work in preparation 


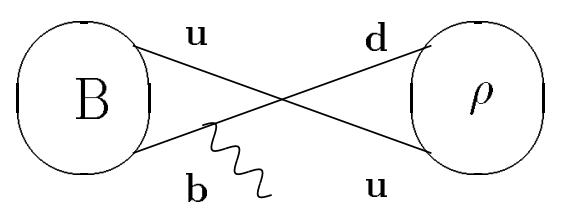

(a)

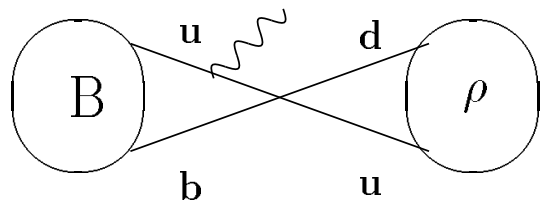

(b)

Figure 1: Weak annihilation mechanism for the decay $B^{-} \rightarrow \rho^{-} \gamma$. Two additional diagrams with photon emission from the final state quarks are not shown.

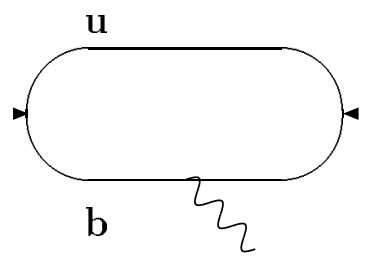

(a)

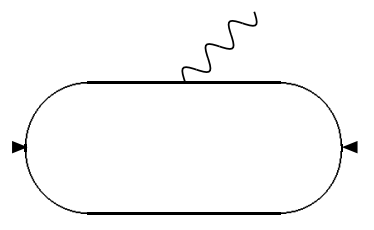

(d)

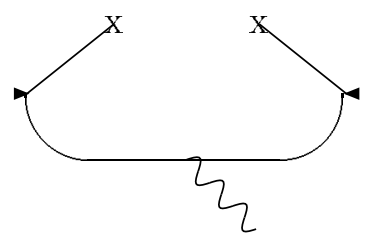

(b)

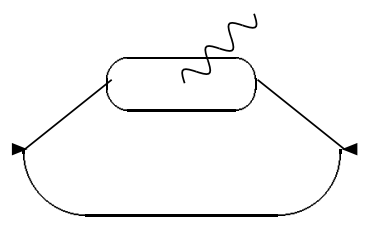

(e)

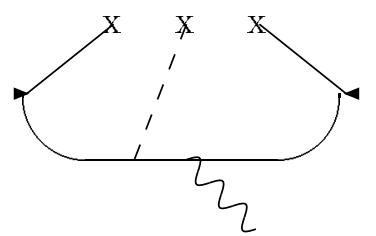

(c)

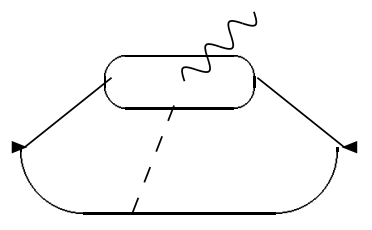

(f)

Figure 2: Diagrams corresponding to OPE of the correlation function in eq.(6): (a), (d) perturbative contributions, (b) quark, (c) quark-gluon condensate contributions; (e) emission of photon at large distances parametrized by photon wave function; ( $f$ ) gluon correction to the diagram (e). Solid lines denote quarks, dashed lines -gluons, wavy line -photon, arrows external currents. Crosses denote vacuum quark and gluon condensates, ovals in the diagrams (e) and (f) denote the photon light-cone wave function. 